\title{
$L$-Stable Block Hybrid Second Derivative Algorithm for Parabolic Partial Differential Equations
}

\author{
Fidele Fouogang Ngwane ${ }^{1 *}$, Samuel Nemsefor Jator ${ }^{2}$ \\ ${ }^{1}$ Department of Mathematics, USC Salkehatchie, Allendale, USA \\ ${ }^{2}$ Department of Mathematics and Statistics, Austin Peay State University, Clarksville, USA \\ Email: ${ }^{\text {fifonge@yahoo.com }}$
}

Received 28 January 2014; revised 28 February 2014; accepted 5 March 2014

Copyright (C) 2014 by authors and Scientific Research Publishing Inc.

This work is licensed under the Creative Commons Attribution International License (CC BY). http://creativecommons.org/licenses/by/4.0/

cC) $\underset{\mathrm{EY}}{\mathrm{B}}$ Open Access

\begin{abstract}
An $L$-stable block method based on hybrid second derivative algorithm (BHSDA) is provided by a continuous second derivative method that is defined for all values of the independent variable and applied to parabolic partial differential equations (PDEs). The use of the BHSDA to solve PDEs is facilitated by the method of lines which involves making an approximation to the space derivatives, and hence reducing the problem to that of solving a time-dependent system of first order initial value ordinary differential equations. The stability properties of the method is examined and some numerical results presented.
\end{abstract}

\section{Keywords}

Hybrid Second Derivative Method; Off-Step Point; Parabolic; Partial Differential Equations

\section{Introduction}

We adopt the method of lines approach which is commonly used for solving time-dependent partial differential equations (PDE), whereby the spatial derivatives are replaced by finite difference approximations (see Lambert [1], Ramos and Vigo-Aguiar [2], Brugnano and Trigiante [3], Cash [4], Enright [5], Hairer et al. [6], Henrici [7], Butcher [8], Fatunla [9], Jator [10], and Onumanyi et al. [11], [12]). Consider the PDE of the form

$$
\frac{\partial u}{\partial t}=\frac{\partial^{2} u}{\partial x^{2}},(x, t) \in[0,1] \times[0<t \leq T]
$$

${ }^{*}$ Corresponding author.

How to cite this paper: Ngwane, F.F. and Jator, S.N. (2014) L-Stable Block Hybrid Second Derivative Algorithm for Parabolic Partial Differential Equations. American Journal of Computational Mathematics, 4, 87-92.

http://dx.doi.org/10.4236/jasmi.2014.42008 
subject to the initial/boundary conditions

$$
u(x, 0)=G(x), x \in[0,1], u(0, t)=u(1, t)=0, t \geq 0 .
$$

We seek a solution in the strip $[0,1] \times[0<t \leq T]$ by first fixing the grid in the spatial variable $x$, then approximating this spatial derivative using the central difference method, and finally solving the resulting system of first order time dependent ODEs. Specifically, we discretize the space variable with mesh spacings $\Delta x=1 / M$,

$$
x_{m}=m \Delta x, m=0,1, \cdots, M .
$$

We then define $u_{m}(t) \approx u\left(x_{m}, t\right), \boldsymbol{u}(t)=\left[u_{1}(t), \cdots, u_{m}(t)\right]^{\mathrm{T}}$, and replace the partial derivatives $\frac{\partial^{2} u(x, t)}{\partial x^{2}}$ occurring in (1) by the central difference approximation to obtain

$\frac{\partial u\left(x_{m}, t\right)}{\partial t}=\left[u\left(x_{m+1}, t\right)-2 u\left(x_{m}, t\right)+u\left(x_{m-1}, t\right)\right] /(\Delta x)^{2} ; m=0,1, \cdots, M-1$, which reduces the PDE to the semidiscrete problem

$$
\frac{\mathrm{d} u_{m}}{\mathrm{~d} t}=\frac{1}{(\Delta x)^{2}}\left(u_{m+1}-2 u_{m}+u_{m-1}\right)
$$

which can be written in the form

$$
\boldsymbol{u}^{\prime}=f(t, \boldsymbol{u}), \boldsymbol{u}(0)=\boldsymbol{u}_{0},
$$

where $\boldsymbol{f}(t, \boldsymbol{u})=\boldsymbol{A u}$, and $\boldsymbol{A}$ is an $M \times M$ matrix arising from the central difference approximations to the derivatives of $x$. The problem (2) is now a system of first order ODEs which is solved by the BHSDA.

The paper is organized as follows. In Section 2, we derive a continuous approximation which is used to obtain the BHSDA. The BHSDA is also analyzed in Section 2. The computational aspects of the method is given in Section 3. Numerical examples are given in Section 4 to show the accuracy of the method. Finally, the conclusion of the paper is discussed in Section 5.

\section{Development of the Method}

We begin by considering a scalar form of (3)

$$
u^{\prime}=f(t, u), u\left(t_{0}\right)=u_{0}, t \in\left[t_{0}, t_{N}\right]
$$

where we assume that the function $\mathrm{f}$ is Lipshitz continuous and the problem (4) possesses a unique solution. Furthermore, let $u_{n}$ be an approximation of the theoretical solution $u(t)$ at $t_{n}$. Our objective is to simultaneously seek numerical approximations at the points $t_{n+v}=t_{n}+v h$ and $t_{n+1}=t_{n}+h$ respectively, where $h$ is the step size, $n$ the grid index, and $v \in(0,1)$. This approximation $u_{n}$ is provided by a continuous approximation $U(t)$ as a by-product. Thus, we assume that $U(t)$ is of the form

$$
U(t)=\sum_{j=0}^{4} \ell_{j} t^{4}
$$

where $\ell_{j}$ are unknown coefficients.

In order to uniquely determine the unknown coefficients $\ell_{j}$, we impose that the interpolating function (4) coincides with the analytical solution at the end point $t_{n}$ and also satisfies the differential Equation (3) at the points $t_{n+j v}, j=0,1,2$ to obtain the following system of equations:

$$
U\left(t_{n}\right)=y_{n}, U^{\prime}\left(t_{n+j v}\right)=f_{n+j v}, U^{\prime \prime}\left(t_{n+1}\right)=g_{n+1}, j=0,1,2 .
$$

We note that (6) leads to a system of five equations which is solved by Cramer's Rule to obtain $\ell_{j}$. The continuous method is constructed by substituting the values of $\ell_{j}$ into Equation (5) which is simplified and expressed in the form

$$
U(t)=y_{n}+h\left(\beta_{0}(t) f_{n}+\beta_{1}(t) f_{n+1}+\beta_{v}(t) f_{n+v}\right)+h^{2} \gamma_{1}(t) g_{n+1}
$$


where $\beta_{0}(t), \quad \beta_{1}(t), \quad \beta_{v}(t), \quad \gamma_{1}(t)$, are continuous coefficients, and $g_{n+1}=\left.\frac{\mathrm{d} f(t, u(t))}{\mathrm{d} t}\right|_{u_{n+1}} ^{t_{n+1}}$. The continuous method (7) is then evaluated at $t=\left\{t_{n+v}, t_{n+1}\right\}$, for $v=1 / 2$ to yield

$$
\left\{\begin{array}{l}
y_{n+1 / 2}=y_{n}+\frac{h}{96}\left(17 f_{n}+44 f_{n+1 / 2}-13 f_{n+1}\right)+\frac{h^{2}}{96}\left(3 g_{n+1}\right) \\
y_{n+1}=y_{n}+\frac{h}{6}\left(f_{n}+4 f_{n+1 / 2}+f_{n+1}\right) .
\end{array}\right.
$$

Remark 2.1 In order to conveniently analyze and implement the method (8), we will express it in block form as given in (9).

$$
A^{(0)} Y_{\mu}=A^{(1)} Y_{\mu-1}+h\left[B^{(0)} F_{\mu}+B^{(1)} F_{\mu-1}\right]+h^{2} C^{(0)} G_{\mu}
$$

where $Y_{\mu}=\left(u_{n+\frac{1}{2}}, u_{n+1}\right)^{\mathrm{T}}, Y_{\mu-1}=\left(u_{n-\frac{1}{2}}, u_{n}\right)^{\mathrm{T}}, F_{\mu}=\left(f_{n+\frac{1}{2}}, f_{n+1}\right)^{\mathrm{T}}, F_{\mu-1}=\left(f_{n-\frac{1}{2}}, f_{n}\right)^{\mathrm{T}}, G_{\mu}=\left(0, g_{n+1}\right)^{\mathrm{T}}$, $\mu=1, \cdots, n=0,1, \cdots$, and the matrices $A^{(0)}, A^{(1)}, B^{(0)}, B^{(1)}, C^{(0)}$ are 2 by 2 matrices whose entries are given by the coefficients of (8).

\subsection{Local Truncation Error}

Define the local truncation error of (4) as

$$
\left\llcorner[z(t) ; h]=Z_{\mu}-A^{(1)} Z_{\mu-1}-h\left[B^{(0)} \bar{F}_{\mu}+B^{(1)} \bar{F}_{\mu-1}\right]-h^{2} C^{(0)} \bar{G}_{\mu}\right.
$$

where

$$
\begin{gathered}
Z_{\mu}=\left(u\left(t_{n+\frac{1}{2}}\right), u\left(t_{n+1}\right)\right)^{\mathrm{T}}, Z_{\mu-1}=\left(u\left(t_{n-\frac{1}{2}}\right), u\left(t_{n}\right)\right)^{\mathrm{T}}, \bar{F}_{\mu}=\left(f\left(t_{n+\frac{1}{2}}, u\left(t_{n+\frac{1}{2}}\right)\right), f\left(t_{n+1}, u\left(t_{n+1}\right)\right)\right)^{\mathrm{T}}, \\
\bar{F}_{\mu-1}=\left(f\left(t_{n-\frac{1}{2}}, u\left(t_{n-\frac{1}{2}}\right)\right), f\left(t_{n}, u\left(t_{n}\right)\right)\right)^{\mathrm{T}} \text {, and } \mathrm{L}[z(t) ; h]=\left(\mathrm{七}_{1}[z(t) ; h], \mathrm{七}_{2}[z(t) ; h]\right)^{\mathrm{T}} \text { is a linear difference }
\end{gathered}
$$

operator. Assuming that $z(t)$ is sufficiently differentiable, we can expand the terms in (10) as a Taylor series about the point $t_{n}$ to obtain the expression for the local truncation error. $E[z(t) ; h]=O\left(h^{5}\right)$, hence the method is of order four.

\subsection{Stability}

Proposition 2.2 The BHSDA (9) applied to the test equations $u^{\prime}=\lambda u$ and $u^{\prime \prime}=\lambda^{2} u$ yields.

$$
Y_{\mu}=M(q) Y_{\mu-1}, q=\lambda h,
$$

with the amplification matrix

$$
M(q)=\left(A^{(0)}-q B^{(0)}-q^{2} C^{(0)}\right)^{-1}\left(A^{(1)}+q B^{(1)}\right)
$$

Remark 2.3 The dominant eigenvalue of $M(q)$ specified by $q_{\max }=\frac{48+18 q+2 q^{2}}{48-30 q+8 q^{2}-q^{3}}$ is a rational function called the stability function which determines the stability of the method.

Proof. We begin by applying (2) to the test equations $u^{\prime}=\lambda u$ and $u^{\prime \prime}=\lambda^{2} u$ which are expressed as $f(t, u)=\lambda u$ and $g(t, u)=\lambda^{2} u$ respectively; letting $q=h \lambda$, we obtain a system of linear equations which is used to solve for $Y_{\mu}$ with (12) as a consequence. 
Definition 2.4 The block method (9) is said to be 1) $A$-stable if for all $q \in \mathbb{C}^{-}, M(q)$ has a dominant eigenvalue $q_{\max }$ such that $\left|q_{\max }\right| \leq 1$; moreover, since $q_{\max }$ is a rational function, the real part of the zeros of $q_{\max }$ must be negative, while the real part of the poles of $q_{\max }$ must be positive; 2$) L$-stable if it is $A$-stable and $q_{\max } \rightarrow 0$ as $q \rightarrow-\infty$.

Corollary 2.5 The method (9) is A-stable and L-stable.

Proof: The dominant eigenvalue $q_{\max }$ for the method (9) is given by $q_{\max }=\frac{48+18 q+2 q^{2}}{48-30 q+8 q^{2}-q^{3}}$ and the proof follows from definition 2.4 .

Remark 2.6 The stability region for the method (9) is given in Figure 1 showing the zeros and poles of the dominant eigenvalue $q_{\max }$.

\section{Computational Aspects}

The resulting system of ODEs (3) is then solved on the partition

$$
\pi_{N}:\left\{t_{0}<t_{1}<\cdots<t_{N}, t_{n}=t_{0}+n h\right\}
$$

$h=\Delta t=\frac{b-a}{N}$ is a constant step-size of the partition of $\pi_{N}, n=1,2, \cdots, N, N$ is a positive integer and $n$ the grid index.

Step 1: Use the block method (9) to solve (3) on rectangles $\left[t_{0}, t_{1}\right] \times[0,1],\left[t_{1}, t_{2}\right] \times[0,1], \cdots,\left[t_{N-1}, t_{N}\right] \times[0,1]$.

Step 2: Let $Y_{m, \mu}=\left(u_{m, n+\frac{1}{2}}, u_{m, n+1}\right)^{\mathrm{T}}$, noting that $u_{m}\left(t_{n}\right) \approx u_{m, n} \approx u\left(x_{m}, t_{n}\right)$, then for $m=1, \cdots, M, n=0$, and $\mu=1$, the approximations $Y_{m, 1}=\left(u_{m, \frac{1}{2}}, u_{m, 1}\right)^{\mathrm{T}}$ are simultaneously obtained on $\left[t_{0}, t_{1}\right] \times[0,1]$.

Step 3: Step 2 is repeated for $m=1, \cdots, M, n=1,2, \cdots, N-1$, and $\mu=2,3, \cdots, N$, to generate the approximations $Y_{m, 2}, Y_{m, 3}, \cdots, Y_{m, N}$ on $\left[t_{1}, t_{2}\right] \times[0,1], \cdots,\left[t_{N-1}, t_{N}\right] \times[0,1]$.

We note that for linear problems, we solve (3) directly with our Mathematica code enhanced by the feature NSolve[ ].

\section{Numerical Examples}

Computations were carried out in Mathematica 9.0 and the errors were calculated as $\left|u_{m, n}-u\left(x_{m}, t_{n}\right)\right|$, where $u_{m}\left(t_{n}\right) \approx u_{m, n}$. We note that the method is particularly useful, but not limited to solving parabolic partial differential equations where the solution decays very rapidly and where the PDEs are stiff parabolic equations (see Cash [4]).

Example 4.1 As our first test example, we solve the given PDE (see Cash [4])

$$
\frac{\partial u}{\partial t}=\kappa \frac{\partial^{2} u}{\partial x^{2}}, u(0, t)=u(1, t)=0, u(x, 0)=\sin \pi x .
$$

The exact solution $u(x, t)=\mathrm{e}^{-\pi^{2} \kappa t} \sin \pi x$.

In Table 1, it is noticed that the method with the BHSDA is the most accurate.

Example 4.2 As our second test example, we solve the given stiff parabolic equation (see Cash [4])

$$
\frac{\partial u}{\partial t}=\kappa \frac{\partial^{2} u}{\partial x^{2}}, u(0, t)=u(1, t)=0, u(x, 0)=\sin \pi x+\sin \omega \pi x, \omega \gg 1 .
$$

The exact solution $u(x, t)=\mathrm{e}^{-\pi^{2} \kappa t} \sin \pi x+\mathrm{e}^{-\omega^{2} \pi^{2} \kappa t} \sin \omega \pi x$.

Cash [4] notes that as $\omega$ increases, equations of the type given in example 4.2 exhibit characteristics similar to model stiff equations. Hence, the methods such as the Crank-Nicolson method which are not $L_{0}$-stable are expected to perform poorly. The BHSDA is $L$-stable and perform excellently when applied to this problem. Therefore the BHSDA is competitive with the $L_{0}$-stable methods of Cash [4]. In Table 2, we display the results for $\kappa=1$ and a range of values for $\omega$. 


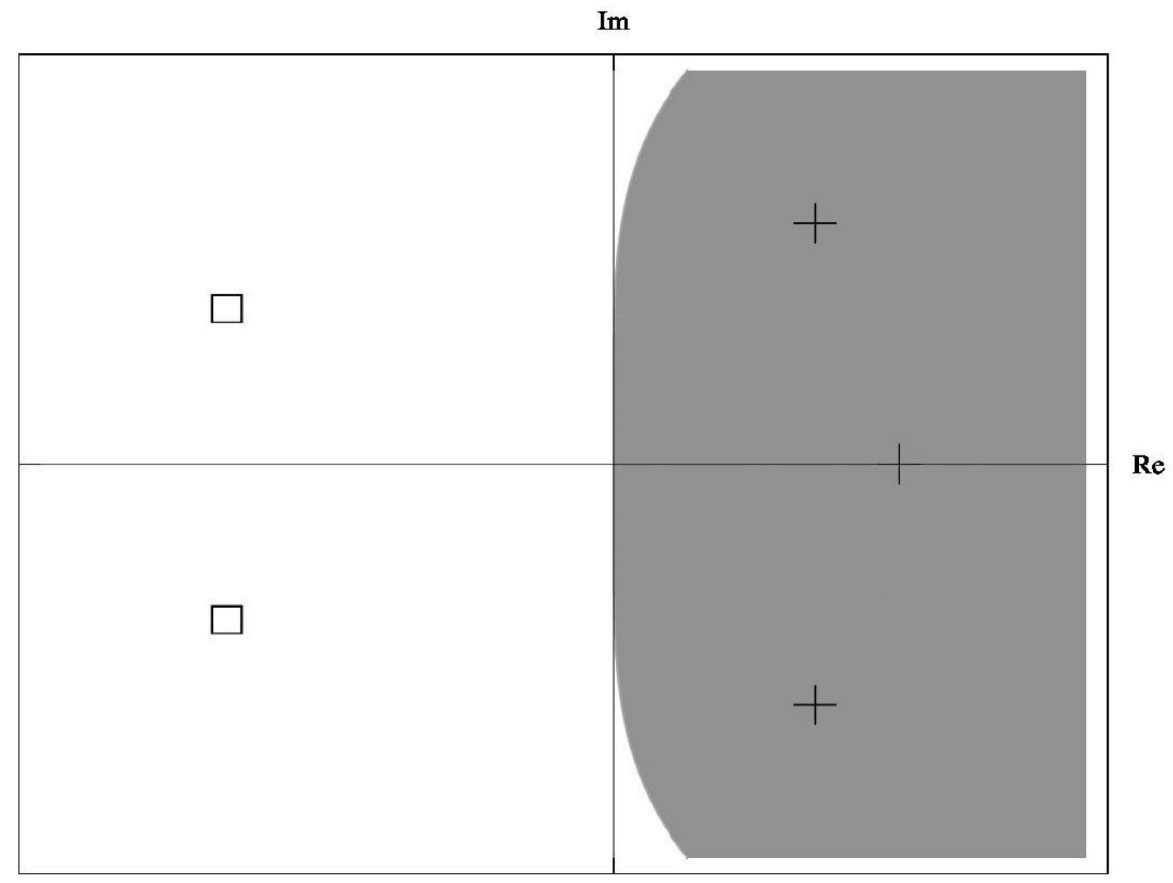

Figure 1. The region of absolute stability of the BHSDA of order 4 is to the left of the dividing line and is symmetric about the real axis; the square and plus symbols to the left and right of the imaginary axis represent the zeros and poles of $q_{\max }$ respectively.

Table 1. A comparison of errors of methods for Example 4.1 at $t=1$.

\begin{tabular}{ccccccc}
$\Delta x$ & $\Delta t$ & $\kappa$ & Crank-Nicolson & Cash (2.6a, b) & Cash (2.13a, b, c) & BHSDA \\
\hline 0.1 & 0.1 & 1 & $3.0 \times 10^{-5}$ & $1.5 \times 10^{-5}$ & $4.5 \times 10^{-6}$ & $1.3 \times 10^{-6}$ \\
0.05 & 0.05 & 1 & $9.0 \times 10^{-6}$ & $4.0 \times 10^{-6}$ & $2.7 \times 10^{-7}$ & $1.7 \times 10^{-7}$ \\
0.1 & 0.1 & 5 & $2.0 \times 10^{-4}$ & $3.0 \times 10^{-8}$ & $2.0 \times 10^{-10}$ & $2.5 \times 10^{-19}$ \\
0.05 & 0.05 & 5 & $1.0 \times 10^{-14}$ & $4.0 \times 10^{-22}$ & $3.7 \times 10^{-22}$ & $7.0 \times 10^{-24}$ \\
\hline
\end{tabular}

Table 2. A comparison of errors of methods for Example 4.1 at $t=1$ and $\omega=1, \Delta x=0.1, \Delta t=0.1$.

\begin{tabular}{|c|c|c|c|c|}
\hline$\omega$ & BHSDA & Crank-Nicolson & Cash (2.6a, b) & Cash (2.13a, b, c) \\
\hline 1 & $2.64 \times 10^{-6}$ & $6.20 \times 10^{-5}$ & $3.7 \times 10^{-5}$ & $1.5 \times 10^{-5}$ \\
\hline 2 & $1.32 \times 10^{-6}$ & $3.83 \times 10^{-5}$ & $1.8 \times 10^{-5}$ & $7.4 \times 10^{-6}$ \\
\hline 3 & $1.32 \times 10^{-6}$ & $9.30 \times 10^{-3}$ & $1.9 \times 10^{-5}$ & $7.4 \times 10^{-6}$ \\
\hline 5 & $1.32 \times 10^{-6}$ & $1.80 \times 10^{-1}$ & $1.8 \times 10^{-5}$ & $7.4 \times 10^{-6}$ \\
\hline 10 & $1.32 \times 10^{-6}$ & $6.10 \times 10^{-1}$ & $1.8 \times 10^{-5}$ & $7.4 \times 10^{-6}$ \\
\hline
\end{tabular}

\section{Conclusion}

We have proposed a BHSDA for solving parabolic PDEs via the method of lines. The method is shown to be $L$ stable and competitive with existing methods in the literature.

\section{References}

[1] Lambert, J.D. (1991) Numerical Methods for Ordinary Differential Systems. John Wiley, New York.

[2] Vigo-Aguiar, J. and Ramos, H. (2007) A family of A-Stable Collocation Methods of Higher Order for Initial-Value Problems. IMA Journal of Numerical Analysis, 27, 798-817. http://dx.doi.org/10.1093/imanum/drl040 
[3] Brugnano, L. and Trigiante, D. (1998) Solving Differential Problems by Multistep Initial and Boundary Value Methods. Gordon and Breach Science Publishers, Amsterdam.

[4] Cash, J.R. (1984) Two New Finite Difference Schemes for Parabolic Equations. SIAM Journal of Numerical Analysis, 21, 433-446. http://dx.doi.org/10.1137/0721032

[5] Enright, W.H. (2000) Continuous Numerical Methods for ODEs with Defect Control. Journal of Computational and Applied Mathematics, 125, 159-170. http://dx.doi.org/10.1016/S0377-0427(00)00466-0

[6] Hairer, E. and Wanner, G. (1996) Solving Ordinary Differential Equations II. Springer, New York. http://dx.doi.org/10.1007/978-3-642-05221-7

[7] Henrici, P. (1962) Discrete Variable Methods in ODEs. John Wiley, New York.

[8] Butcher, J.C. (1987) The Numerical Analysis of Ordinary Differential Equations, Runge-Kutta and General Linear Methods. Wiley, New York.

[9] Fatunla, S.O. (1991) Block Methods for Second Order IVPs. International Journal of Computational Mathematics, 41, 55-63. http://dx.doi.org/10.1080/00207169108804026

[10] Jator, S.N. (2010) On the Hybrid Method with Three-Off Step Points for Initial Value Problems. International Journal of Mathematical Education in Science and Technology, 41, 110-118 http://dx.doi.org/10.1080/00207390903189203

[11] Onumanyi, P., Sirisena, U.W. and Jator, S.N. (1999) Continuous Finite Difference Approximations for Solving Differential Equations. International Journal of Computational Mathematics, 72, $15-27$. http://dx.doi.org/10.1080/00207169908804831

[12] Onumanyi, P., Awoyemi, D.O., Jator, S.N. and Sirisena, U.W. (1994) New Linear Mutlistep Methods with Continuous Coefficients for First Order Initial Value Problems. Journal of the Nigerian Mathematics Society, 37-51. 